\title{
ROUNDUP360
}

Wrist \& Hand

Releasing the trigger finger

n Trigger fingers seem fairly common and will often come under generalists as well as subspecialist hand surgeons. Yet how does an open release of trigger finger fare? 360 finds a paper from Bad Rappenau (Germany) interesting in this respect. Researchers looked at 117 releases of trigger fingers in 103 patients with the aim of identifying the post-operative complications and disorders associated with the procedure. Factors that were investigated included demographic details, the number of digits affected, body mass index (BMI), level of manual strain, trauma, medication, hand dominance, pre-treatment with steroid injection, and concomitant diseases. The results showed that the dominant hand was not more frequently affected than the nondominant side, nor did occupation influence the incidence of trigger finger. Meanwhile, patients receiving systemic steroid therapy had a shorter duration of post-operative symptoms, with a mean duration of 29.3 days. Interestingly, less post-operative swelling was noticed in patients who had received a presurgical steroid injection. The mean duration of symptoms before and after surgery was shorter for a trigger thumb than for trigger finger. ${ }^{1} 360$ agrees with the authors that an open release appears a suitable low-risk surgical procedure for treatment of trigger finger. Additionally, this study illustrates the importance of careful post-operative follow-up treat- ment to avoid persistent functional limitations. This latter conclusion is important for those countries where healthcare management is keen for patients to be discharged from all care at the earliest opportunity.

\section{Function in the} osteoarthritic hand

- Osteoarthritis (OA) in the hand can be enormously debilitating and greatly limit the performance of everyday activities. Yet what are the practical effects of these limitations? These have in part been investigated by workers from Santa Catarina (Brazil) who assessed grip force control in individuals with $\mathrm{OA}$ of the hand during the lifting of an object. Through a case-control study they compared ten women with OA of the hand with a group of age-matched women without the condition and asked them to perform two functional tasks. One was to lift an instrumented object vertically while the load was suddenly changed and the other was to lift and place the object on a shelf. Load Force Peak, Grip Force at liftoff, Grip Force Peak, Time Lag, and Latency were measured. Those individuals with $\mathrm{OA}$ of the hand were able to modulate the magnitude and temporal parameters of grip force; however, they applied higher grip forces and demonstrated a longer latency to manipulate the instrumented object when compared with controls. ${ }^{2} 360$ wonders what the explanation of these findings might be; lack of confidence in the function of a painful hand, perhaps? Whatever the reasons, detailed information about how individuals with OA of the hand perform manual activities of daily living will help to better understand the limitations of grip force control that they demonstrate.

\section{Complex regional pain syndrome}

- A known complication of surgery or trauma to the upper limb and hand is complex regional pain syndrome. It can be very difficult to treat. A simple and easily tolerated method of treatment employed by some centres is an intravenous regional anaesthetic block with lidocaine (lignocaine, Xylocaine) and methylprednisolone. A team presents such treatment from Larissa (Greece). Here, 168 patients with complex regional pain syndrome of the upper limb were treated over a five-year period. The results were pleasing, with $88 \%$ of the patients reporting little or no pain by the end of treatment. However, it appears that things continued to improve as, after a mean follow-up of five years, $92 \%$ of patients reported a complete absence of pain. Furthermore, the symptoms of the acute phase of the condition were reversed. ${ }^{3} 360$ notes that the authors feel early recognition and prompt treatment is important for the course of the disease as symptoms can be reversible when treatment starts early. Sitting tight to see how the situation develops is clearly not the best recommendation for complex regional pain syndrome in the upper limb and hand.

Arthroscopic ligamentoplasty

\section{for the injured scapholunate ligament}

- With many new surgical techniques, a period spent in a cadaver laboratory is time well spent. Work from Madrid (Spain) highlights this, where surgeons present an anatomical study and description of a new surgical technique for arthroscopic treatment of injuries to the scapholunate ligament. They used five cadaver specimens to perform an arthroscopic ligamentoplasty. After arthroscopic surgery, an anatomical dissection was performed to measure the distances to critical wrist structures such as the posterior interosseous nerve and the radial artery, and the size and position of their repair. The workers conclude that their arthroscopic technique offers three advantages: soft-tissue damage is reduced (avoiding an extensive approach and injury to the secondary stabilisers and reducing scar tissue); injury to the posterior interosseous nerve is avoided (maintaining wrist proprioception and the role of the dynamic stabilisers); and a biotenodesis is made that ensures proper placement, tension and function of the ligament reconstruction. ${ }^{4} 360$ feels that we can all learn from this paper. Certainly more of us should spend time in a cadaver laboratory before embarking on new and novel techniques.

\section{Self-concept and upper limb} deformities in children

- The resilience of children in the face of adversity has long impressed the editorial team at 360 . What 
worries a child may not worry an adult, while something that troubles an adult may not perturb a child at all. Researchers from Gothenburg (Sweden) have undertaken a fascinating study into self-concept and psychological well-being in children with hand deformities and upper limb deficiencies. They included 92 children ( 53 boys, 39 girls) between the ages of nine and 11 years. The children were divided into two subgroups - one with milder, less visible deformities and one with severe and highly visible finger-handarm deformities. The Piers-Harris Children's Self-Concept Scale, where higher scores are good, was used to measure self-esteem and wellbeing. Astonishingly, the wholehand deformity group had 'good' self-concept with mean scores equal to a comparison group of healthy children. Meanwhile those with mild deformities had lower scores than those with severe ones. To cap it all, the children with severe deformities had even higher scores than the comparison group in the subscale for 'Intellectual and School Status'. The children with milder deformities had lower scores than the comparison group in the subscale 'Popularity'. ${ }^{5}$ Astonishing stuff, thinks 360. Children will continue to surprise us all.

\section{Wrist arthroscopy in children}

- Children also feature in a paper on wrist arthroscopy presented by a team from Vienna (Austria). Here, a single-surgeon series of 34 wrist arthroscopies in children and adolescents with chronic wrist pain is reported. There were 27 girls and six boys with a mean age at the time of arthroscopic exploration of 14.6 years. A total of 28 ( $82.4 \%)$ wrists showed a tear of the triangular fibrocartilage complex (TFCC). In $26(76.5 \%)$ of the 34 procedures further pathology was seen, in addition to the TFCC tears. A number of arthroscopic procedures were performed, including 14 arthroscopic resections of the TFCC, two arthroscopic repairs of the TFCC and seven open resection arthroplasties. Other concomitant procedures (e.g. ulnar shortening) were performed in 25 patients ( 26 wrists) because of predisposing deformities. ${ }^{6}$ Pleasingly, 360 notes that no intra-operative or post-operative complications were encountered and no relevant technical differences between a wrist arthroscopy in an adult and that in a child were experienced. The authors do highlight, however, something with which 360 most certainly agrees; this procedure should only be performed by well-trained and experienced hand surgeons.

\section{Internal or external}

\section{fixation for the fractured} distal radius?

- From Ontario (Canada) comes an interesting study into the surgical management of the fractured distal radius. If you intend to operate should you internally fix or percutaneously pin with external fixation? The authors randomised 53 patients with fractures of the distal radius, who had experienced a failed closed reduction, to either open reduction and internal fixation (ORIF) or external fixation.

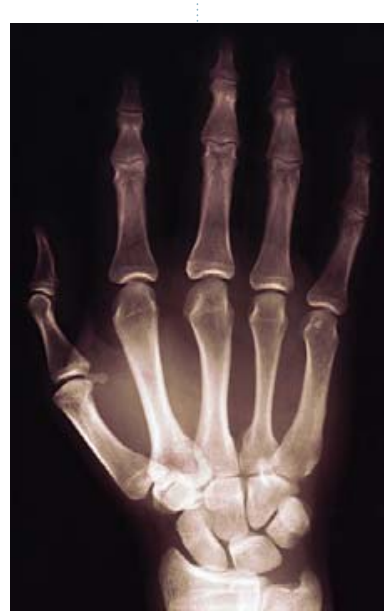

surgery but at six weeks and three months afterwards. The PRWE scores were significantly lower for patients treated with ORIF compared with those who received external fixation, the best outcomes being seen with volar locking plates. A higher mean initial pre-operative PRWE score was seen with external fixation, perhaps indicating a more severe initial injury. 360 shares the authors' puzzlement when they report that their interpretation of the results is unclear. This is actually quite a refreshing conclusion in an era when so many publications seem to report positive conclusions.

\section{Nerve grafting - double or} single end-to-side?

- When grafting a nerve, should you use a single or double nerve graft? Laboratory work from Chiba (Japan) has attempted to answer this. The researchers wished to establish whether an end-to-side double nerve graft displayed more axonal regeneration than a single nerve graft in a rat's lower limb. To do this, they used the lower limbs of 96 Wister rats. Left peroneal nerves were harvested and grafted between the right peroneal and tibial

For pragmatic reasons, the choice of ORIF was left to the surgeon's discretion. Outcomes were measured before surgery, and at six weeks, three, six and 12 months after the procedure. Outcome measures included the Patient-Rated Wrist Evaluation (PRWE - lower scores are good), Disabilities of the Arm, Shoulder, and Hand (DASH) scores, as well as range of movement, grip strength and serial radiographic analysis. Overall, the ORIF group scored 11 points lower on the PRWE across all time points compared with the external fixation group. There was also higher pain and disability associated with external fixation, not only before of motor and sensory nerve fibres was possible using two end-to-side nerve grafts. However, there was no significant difference between single and double grafts. This might suggest a therapeutic limitation of nerve transplants using two end-to-side nerve grafts. Double end-to-side repair attracts both motor and sensory axons, and this results in a medium degree of recovery of function; however, double end-to-side nerve grafting does not appear to offer any advantage over a single end-to-side graft. ${ }^{8}$

\section{Splinting the PIPJ contracture}

- To eliminate a joint contracture using an orthotic device has always struck 360 as an imprecise science, so a study from Brisbane (Australia) is of interest. The authors wished to establish if there was a relationship between the resolution of a contracture in a stiff proximal interphalangeal joint (PIPJ) and the length of time a dynamic orthoses was applied for both flexion and extension deficits of the joint. They treated 41 participants with a dynamic orthotic device (splint) for either a flexion or extension deficit of the PIPJ. The relationship between the resolution of the contracture and the length of treatment was then examined. The researchers found that the outcome with orthotic use was significantly associated with the length of treatment. The range of movement increased in a linear fashion although faster progress was observed when treatment was aimed at improving flexion rather than extension. Flexion deficits appeared to maximise gains with orthotic treatment after 12 weeks. Meanwhile, extension deficits continued to demonstrate slow and continuous improvement beyond the 17 weeks of recorded data. ${ }^{9} 360$ feels these findings will be particularly helpful when explaining to patients why they have to wear their splint for what appears to be so long.

\section{Finding the stalk of a dorsal wrist ganglion}

- Occasionally technical papers appear which can be of value not 
just to subspecialist hand surgeons but also to us lesser mortals, too. Consequently, a short report presented by surgeons from Providence (USA) appeals to 360 . They discuss the treatment of the dorsal wrist ganglion, one of the most common soft-tissue masses of the hand and wrist. Over the past ten to 15 years, they write, there has been a growing interest in arthroscopic treatment of these lesions. Proposed advantages of arthroscopy include greater movement, improved cosmesis, and the potential to identify and treat other intra-articular pathology. However, a limitation of this arthroscopic technique is an inconsistent identification of the ganglion stalk. To make this easier, during the proce- dure the surgeons inject a small volume of methylene blue into the cyst. Its communication with the joint is apparent arthroscopically, thus identifying the location of the stalk. ${ }^{10}$ How simple! 360 wishes they had thought of this in the days when these ganglia were being excised openly. The authors note that the use of methylene blue should result in fewer recurrences, decreased operative time, and less iatrogenic injury.

\section{REFERENCES}

1. Cakmak F, Wolf MB, Bruckner T, Hahn P, Unglaub $F$. Follow-up investigation of open trigger digit release. Arch Orthop Trauma Surg 2011;(Epub ahead of print) PMID:22160513.

2. de Oliveira DG, Nunes PM, Aruin AS, Dos Santos MJ. Grip force control in individuals with hand osteoarthritis. J Hand Ther 2011;24:345-354.

3. Varitimidis SE, Papatheodorou LK, Dailiana H, Poultsides L, Malizos KN. Complex regional pain syndrome type I as a consequence of trauma or surgery to upper extremity: management with intravenous regional anaesthesia, using lidocaine and methyloprednisolone. J Hand Surg Eur Vol 2011;36:771-777.

4. Corella F, Del Cerro M, LarrainzarGarijo R, Vázquez T. Arthroscopic ligamentoplasty (bone-tendon-tenodesis): a new surgical technique for scapholunate instability: preliminary cadaver study. I Hand Surg Eur Vol 2011;36:682-689.

5. Andersson GB, Gillberg C, Fernell $E$, Johansson M, Nachemson A. Children with surgically corrected hand deformities and upper limb deficiencies: self-concept and psychological well-being. J Hand Surg Eur Vol 2011;36:795-801.

6. Farr S, Grill F, Girsch W. Wrist arthroscopy in children and adolescents: a single surgeon experience of thirty-four cases. Int Orthop 2011;(Epub ahead of print) PMID:22159549.

7. Grewal R, MacDermid JC, King GJ,

Faber KJ. Open reduction internal fixation versus percutaneous pinning with external fixation of distal radius fractures: a prospective, randomized clinical trial. J Hand Surg Am 2011;36:1899-1906.

8. Iwakura $\mathrm{N}$, Ohtori $\mathrm{S}$, Kenmoku $\mathrm{T}$, et al. Single versus double end-to-side nerve grafts in rats. J Hand Surg Am 2011;37:261-259.

9. Glasgow C, Fleming J, Tooth LR, Hockey RL. The long-term relationship between duration of treatment and contracture resolution using dynamic orthotic devices for the stiff proximal interphalangeal joint: a prospective cohort study. J Hand Ther 2011;25:38-47.

10. Lee BJ, Sawyer GA, Dasilva MF. Methylene blue-enhanced arthroscopic resection of dorsal wrist ganglions. Tech Hand Up Extrem Surg 2011;15:243-246. 